\title{
Prevalencia al nacimiento de malformaciones congénitas en hijos de madres adolescentes. Hospital Clínico Universidad de Chile. Período 2002-2011
}

\author{
JULIO NAZER H. ${ }^{1}$, LUCÍA CIFUENTES O. ${ }^{2}$
}

'Departamento de Obstetricia y Ginecología. Universidad de Chile. 2Programa de Genética Humana. Instituto de Ciencias Biomédicas Facultad de Medicina. Universidad de Chile.

Recibido el 10 de diciembre de 2012, aceptado el 1 de agosto de 2013.

Correspondencia a: Dr. Julio Nazer Herrera. Departamento de Obstetricia y Ginecología Universidad de Chile. Santiago-Chile

E-mail:jnazer@hcuch.cl

\section{Congenital malformations among newborns of teenage mothers}

Background: The Chilean Ministry of Health has reported a meaningful increase of births from teenager mothers (aged less than 20 years) in the period 1990-2008. On the contrary, there was a decrease of births from teenage mothers at The University of Chile Clinical Hospital (HCUCH). Aim: To compare the prevalence rates at birth of congenital malformations (CMF) in newborns from mothers younger than 20 with those of mothers between 20 and 34 years old. Patients and Methods: The Latin American Collaborative Study of Congenital Malformations (ECLAMC) data base of the HCUCH, since 1969 was analyzed. The last ten years (2002-2011) were studied. Results: There were 15,636 births in the period studied, 153 of them were stillbirths $(0.97 \%)$. There were 1174 newborns from teenage mothers, 82 of them had one or more congenital malformations (7\%). We found an association between smoking and drug consumption in mothers and CMF in their newborns. Conclusions: The rate of congenital malformations among offspring of teenage mothers is lower than the rate for mothers between 20 and 34 years old.

(Rev Med Chile 2013; 141: 1300-1306).

Key words: Malformations congenital, teenager mothers; Pregnancy in adolescence; Teenagers.
S egún informe del Ministerio de Salud de Chile (MINSAL) del período 1990-2008 ha habido un aumento significativo de los nacimientos de hijos de madres adolescentes en el país (Tabla 1). En efecto, en 1990, 13,8\% de los nacimientos fueron de mujeres menores de 20 años, cifra que aumentó en el año 2008 a 16,6\%, lo que representa un incremento de $20,3 \%$. Significa que alrededor de 40.000 nacimientos al año corresponden a hijos de mujeres adolescentes. Sin embargo, esto no ocurre en el Hospital Clínico de la Universidad de Chile ( $\mathrm{HCUCH})$ donde hubo una reducción de $34 \%$ de la proporción de madres adolescentes en el período 1982-2011, de 16,8\% en el año 1982 a 7,50\% en el 2011 (Tabla 2).
Hay varias situaciones de este grupo etario que es necesario estudiar en nuestro medio, ya que son potencialmente factores de riesgo para malformaciones congénitas (MFC), especialmente hábitos o forma de vida, escolaridad y nivel socioeconómico.

Algunas publicaciones extranjeras han comunicado mayor prevalencia al nacimiento de $\mathrm{MFC}^{3,4}$ de partos prematuros y bajo peso de nacimiento en los hijos de madres adolescentes ${ }^{5,6}$.

Según nuestros datos publicados en $2003^{7}$ para el período 1982-2001, no encontramos mayor frecuencia de malformaciones congénitas, bajo peso de nacimiento ni de prematuridad en sus hijos.

Los objetivos del presente trabajo son verificar 
Malformaciones congénitas en hijos de madres adolescentes - J. Nazer et al

Tabla 1. Distribución de los nacimientos según grupos de edades maternas en Chile. Período 1990-2008

\begin{tabular}{|c|c|c|c|c|c|c|c|}
\hline \multirow[t]{2}{*}{ Año } & \multirow{2}{*}{$\begin{array}{c}\text { Total } \\
\text { nacimientos }\end{array}$} & \multicolumn{6}{|c|}{ Edad de la madre } \\
\hline & & $<$ de 20 años & $\%$ & 20 a 34 años & $\%$ & $>$ de 34 años & $\%$ \\
\hline 1990 & 292.145 & 40.285 & 13,8 & 222.569 & 76,2 & 29.291 & 10,0 \\
\hline 1991 & 284.482 & 39.030 & 13,7 & 214.738 & 75,5 & 30.714 & 10,8 \\
\hline 1992 & 279.098 & 38.483 & 13,8 & 209.366 & 75,0 & 31.249 & 11,2 \\
\hline 1993 & 275.916 & 38.555 & 14,0 & 205.578 & 74,5 & 31.912 & 11,6 \\
\hline 1994 & 273.766 & 38.848 & 14,2 & 202.740 & 74,1 & 32.178 & 11,8 \\
\hline 1995 & 265.932 & 38.885 & 14,6 & 194.332 & 73,4 & 32.715 & 12,3 \\
\hline 1996 & 264.793 & 39.701 & 15,0 & 191.255 & 72,2 & 33.837 & 12,8 \\
\hline 1997 & 259.959 & 40.734 & 15,7 & 184.715 & 71,1 & 34510 & 13,3 \\
\hline 1998 & 257.105 & 41.530 & 16,2 & 180.568 & 70,2 & 35.007 & 10,2 \\
\hline 1999 & 250.674 & 40.439 & 16,1 & 174.514 & 69,6 & 35.721 & 14,2 \\
\hline 2000 & 248.893 & 40.312 & 16,2 & 171.628 & 69,0 & 36.953 & 14,8 \\
\hline 2001 & 246.116 & 39.884 & 16,2 & 168.278 & 68,4 & 37.954 & 15,4 \\
\hline 2002 & 238.981 & 37.618 & 15,7 & 163.764 & 68,5 & 37.599 & 15,7 \\
\hline 2003 & 234.486 & 34.832 & 14,9 & 161.536 & 68,9 & 38.118 & 16,3 \\
\hline 2004 & 230.831 & 34.424 & 14,9 & 158.264 & 68,7 & 37.664 & 16,3 \\
\hline 2005 & 230.831 & 36.069 & 15,6 & 157.290 & 68,1 & 37.472 & 16,2 \\
\hline 2006 & 231.383 & 37.773 & 16,3 & 156.034 & 67,4 & 37.576 & 16,2 \\
\hline 2007 & 240.569 & 39.605 & 16,5 & 162.754 & 67,7 & 38.165 & 15,7 \\
\hline 2008 & 246.581 & 40.927 & 16,6 & 166.644 & 67,6 & 38.977 & 15,8 \\
\hline
\end{tabular}

*Datos proporcionados por Ministerio de Salud de Chile.

en el HCUCH la tendencia de las edades maternas bajas a disminuir en porcentaje respecto a otros grupos etarios, obtener las tasas de prevalencia de MFC en los hijos de madres adolescentes. Comparar estas tasas con las del grupo de hijos de madres de 20 a 34 años. Investigar la relación que pudiera haber entre la prevalencia de MFC y los hábitos de vida, como consumo de alcohol, tabaco y drogas, en este grupo etario. Comparar algunos factores demográficos de este grupo con el grupo control, como prematuridad, bajo peso de nacimiento, mortinatalidad, mortalidad neonatal, RCIU.

\section{Pacientes y Método}

Se analizó la base de datos que el ECLAMC (Estudio Colaborativo Latino Americano de Malfor- maciones Congénitas) tiene en el HCUCH desde 1969. A ella ingresan todos los recién nacidos (RN), vivos y mortinatos con peso de nacimiento de 500 g o más y que presenten una o más malformaciones congénitas. Se tomó como grupo control a los hijos de las madres entre 20 y 34 años de edad. Los malformados nacidos muertos no tienen control ${ }^{8}$.

Se distribuyó los nacimientos en tres grupos según la edad materna, menores de 20 años, entre 20 y 34 años y mayores de 34 años (Tabla 3 ).

Se estudia el período de 10 años comprendido entre el 1 de enero de 2002 y 31 de diciembre de 2011. Anteriormente publicamos el período 1982-20017.

El estudio estadístico se realizó comparando proporciones con prueba $\chi^{2}$ o test exacto de Fisher en las muestras pequeñas y con prueba t de Student para la comparación de variables cuantitativas. 


\section{Resultados}

La distribución por años de los nacimientos según los grupos etarios maternos estudiados, menores de 20 años, entre 20 y 34 años y mayores de 34 años, se muestran en la Tabla 3. En el período

\section{Tabla 2. Distribución porcentual de edades maternas por grupos. \\ Hospital Clínico Universidad de Chile}

\begin{tabular}{|c|c|c|c|c|}
\hline Año & $<20$ años & 20-34 años & $>34$ años & \\
\hline 1982 & 16,08 & 74,12 & 9,8 & 100 \\
\hline 1983 & 13,56 & 76,96 & 9,48 & 100 \\
\hline 1984 & 13,86 & 74,90 & 11,24 & 100 \\
\hline 1985 & 12,48 & 77,27 & 10,25 & 100 \\
\hline 1986 & 11,97 & 78,48 & 9,55 & 100 \\
\hline 1987 & 7,92 & 80,58 & 11,50 & 100 \\
\hline 1988 & 7,33 & 80,65 & 12,02 & 100 \\
\hline 1989 & 8,05 & 80,64 & 11,31 & 100 \\
\hline 1990 & 11,50 & 77,37 & 11,13 & 100 \\
\hline 1991 & 12,69 & 76,56 & 10,75 & 100 \\
\hline 1992 & 13,17 & 75,59 & 11,24 & 100 \\
\hline 1993 & 12,98 & 73,52 & 13,50 & 100 \\
\hline 1994 & 11,09 & 75,86 & 13,05 & 100 \\
\hline 1995 & 4,11 & 82,08 & 13,81 & 100 \\
\hline 1996 & 4,40 & 81,67 & 13,93 & 100 \\
\hline 1997 & 5,68 & 79,56 & 14,76 & 100 \\
\hline 1998 & 6,24 & 77,92 & 15,84 & 100 \\
\hline 1999 & 5,87 & 75,55 & 18,58 & 100 \\
\hline 2000 & 5,93 & 74,46 & 19,61 & 100 \\
\hline 2001 & 6,14 & 73,46 & 20,40 & 100 \\
\hline 2002 & 5,93 & 74,02 & 20,05 & 100 \\
\hline 2003 & 4,92 & 72,31 & 22,77 & 100 \\
\hline 2004 & 4,61 & 72,06 & 23,33 & 100 \\
\hline 2005 & 6,70 & 71,85 & 21,45 & 100 \\
\hline 2006 & 8,16 & 69,39 & 22,45 & 100 \\
\hline 2007 & 7,62 & 71,98 & 20,40 & 100 \\
\hline 2008 & 10,51 & 70,30 & 19,19 & 100 \\
\hline 2009 & 8,47 & 69,09 & 22,44 & 100 \\
\hline 2010 & 9,09 & 68,74 & 22,16 & 100 \\
\hline 2011 & 7,50 & 70,75 & 21,74 & 100 \\
\hline
\end{tabular}

ocurrieron 15.636 nacimientos consecutivos de los que 15.483 fueron nacidos vivos (NV) y 153 mortinatos. Esto representa una mortinatalidad o mortalidad fetal tardía de 0,98\%.

En esta muestra se encontraron $1.174 \mathrm{RN}$ hijos de madres menores de 20 años (7,5\%; 1.174/15.636). De ellos 1.162 eran nacidos vivos $(7,4 \% ; 1.162 / 15.636)$ y 12 mortinatos $(7,8 \%$; $12 / 153)$; mortinatalidad semejante a aquella observada en hijos de madres entre 20 y 34 años $(\mathrm{p}=0,68)$. Tres mil trescientos noventa y nueve RN (21,7\%; 3.399/15.636) eran hijos de mujeres mayores de 34 años, de ellos 3.358 fueron NV $(21,4 \% ; 3.358 / 15.636)$ y 41 mortinatos $(26,8 \%$; $41 / 153)$. En el grupo de hijos mujeres de 20 a 34 años hubo $11.063 \mathrm{RN}$ distribuidos de la siguiente manera: $10.963 \mathrm{NV}(70 \%$; $10.063 / 15.636)$ y 100 mortinatos $(0,9 \%)$ (Tabla 3$)$.

En la Tabla 4 se compara algunos factores demográficos de los hijos malformados de madres adolescentes con sus controles no malformados. Se puede apreciar que hay diferencias significativas sólo en consumo de drogas y tabaquismo. El resto de las diferencias no alcanzaron significación estadística. Entre los hijos de madres adolescentes, $19 \mathrm{RN}$ tenía menos de 38 semanas de gestación al nacer $(23,2 \%)$ y $20 \mathrm{RN}$ pesaron menos de $2.500 \mathrm{~g}$ $(24,4 \%), 34,1 \%$ de sus madres refería ingerir alcohol, en tanto que $64 \mathrm{RN}$ eran adecuados a la edad gestacional (AEG) (78\%), $13 \mathrm{RN}$ eran pequeños para la edad gestacional (PEG) $(15,9 \%)$ y $5 \mathrm{RN}$ eran grandes para la edad gestacional (GEG) $(6,1 \%)$. Treinta madres adolescentes tenían el antecedente de otro malformado en la familia $(36,5 \%)$ y no había casos de consanguinidad.

Respecto de los antecedentes educacionales, 12 madres tenían estudios universitarios incompletos (14,6\%), 18 tenían estudios secundarios completos (22\%), 48 con secundaria incompleta $(58,5 \%), 3$ con primaria completa $(3,7 \%)$ y 1 con primaria incompleta $(1,2 \%)$.

En el grupo de hijos de adolescentes se encontró $82 \mathrm{RN}$ que presentaban una o más malformaciones congénitas (7\%), 81 eran NV y sólo $1 \mathrm{NM}$ $(1,2 \%)$. Esta prevalencia es $25,5 \%$ menor ( $\mathrm{p}=$ $0,009)$ comparada con $9,6 \%$ encontrada en el estudio previo efectuado para el período 1982-2001.

La Tabla 5 muestra la distribución de malformaciones congénitas por sistema, donde las anomalías de piel y fanéreos, esqueléticas y cardiovasculares son las más frecuentes. 
Malformaciones congénitas en hijos de madres adolescentes - J. Nazer et al

Tabla 3. Distribución por año de los nacimientos por grupos de edades maternas. Total de nacimientos 15.636. Hospital Clínico de la Universidad de Chile. Período 2002-2011

\begin{tabular}{|c|c|c|c|c|c|c|c|c|c|c|}
\hline \multirow[t]{2}{*}{ Año } & \multicolumn{3}{|c|}{$<$ de 20 años } & \multicolumn{3}{|c|}{ de 20-34 años } & \multicolumn{3}{|c|}{ > de 34 años } & \multirow[t]{2}{*}{ Total } \\
\hline & NV & NM & Total & NV & NM & Total & NV & NM & Total & \\
\hline 2002 & 109 & 2 & 111 & 1.362 & 12 & 1.374 & 369 & 6 & 375 & 1.890 \\
\hline 2003 & 89 & 2 & 91 & 1.230 & 8 & 1.238 & 389 & 3 & 392 & 1.721 \\
\hline 2004 & 90 & 0 & 90 & 1.126 & 13 & 1.139 & 322 & 3 & 325 & 1.554 \\
\hline 2005 & 101 & 0 & 101 & 1.082 & 11 & 1.093 & 323 & 5 & 328 & 1.522 \\
\hline 2006 & 121 & 1 & 122 & 1.034 & 6 & 1.040 & 333 & 2 & 335 & 1.497 \\
\hline 2007 & 120 & 1 & 121 & 1.127 & 10 & 1.137 & 340 & 2 & 342 & 1.600 \\
\hline 2008 & 172 & 0 & 172 & 1.137 & 12 & 1.149 & 314 & 6 & 320 & 1.641 \\
\hline 2009 & 125 & 3 & 128 & 1.019 & 9 & 1.028 & 331 & 5 & 336 & 1.492 \\
\hline 2010 & 129 & 0 & 129 & 979 & 11 & 990 & 317 & 5 & 322 & 1.441 \\
\hline 2011 & 106 & 3 & 109 & 867 & 8 & 875 & 320 & 4 & 324 & 1.305 \\
\hline Total & 1.162 & 12 & 1.174 & 10.963 & 100 & 11.063 & 3.358 & 41 & 3.399 & 15.636 \\
\hline
\end{tabular}

Tabla 4. Comparación entre los hijos malformados de madres menores de 20 años y sus controles sanos. Hospital Clínico de la Universidad de Chile. Período 2002-2011

\begin{tabular}{|c|c|c|c|}
\hline \multirow[b]{2}{*}{ Peso nacimiento $(\mathrm{g})$} & \multicolumn{2}{|c|}{$\begin{array}{cc} & \text { Malfomados } \\
\text { Hijos de madres }<20 \text { años } & \text { Controles } \\
\text { n: } 82 & \text { n: } 81\end{array}$} & \multirow{2}{*}{$\mathbf{p}$} \\
\hline & $X=3.073,4 \quad($ d.s. $=764,6)$ & $X=3.229,7 \quad($ d.s. $=690,2)$ & \\
\hline$\%$ nacidos vivos & 98,7 & 100 & 0,320 \\
\hline$\%$ mortinatos & 1,2 & 0 & 0,320 \\
\hline$\%$ mortineonatos & 1,2 & 0 & 0,320 \\
\hline$\%$ PEG & 15,9 & 7,9 & 0,370 \\
\hline Edad gestacional (sem.) & $X=37,7 \quad($ d.s. $=3,3)$ & $38,0 \quad($ d.s. $=3,0)$ & 0,630 \\
\hline Tabaquismo \% & 39 & 22,2 & 0,004 \\
\hline Droga \% (marihuana) & 18,3 & 0,0 & 0,001 \\
\hline$\%$ bajo peso & 24,4 & 16,3 & 0,120 \\
\hline$\%$ prematuros & 23,2 & 25,5 & 0,880 \\
\hline Consumo de alcohol \% & 34,1 & 37,0 & 0,690 \\
\hline Consumo de tabaco $\%$ & 39,0 & 22,0 & 0,100 \\
\hline Metrorragia \% & 6,1 & 1,2 & 0,100 \\
\hline
\end{tabular}

$\mathrm{X}=$ promedio; d.s. = desviación standard.

Tabla 5. Distribución de las malformaciones por sistemas

\begin{tabular}{|lcc|}
\hline Sistema & n de malformaciones & $\%$ \\
\hline Piel y fanerios & 30 & 34,3 \\
\hline Esqueléticas & 17 & 18,9 \\
Cardiovasculares & 10 & 11,1 \\
\hline Digestivas & 6 & 6,7 \\
Renourinarias & 4 & 4,3 \\
Sistema nervioso central & 4 & 4,3 \\
Glándulas de secreción interna & 4 & 4,3 \\
Órganos de los sentidos & 4 & 4,3 \\
Cromosómicas & 3 & 3,3 \\
Otras & 8 & 8,5 \\
\hline Total & 90 & 100 \\
\hline
\end{tabular}


Malformaciones congénitas en hijos de madres adolescentes - J. Nazer et al

Tabla 6 a. Comparación de las tasas de prevalencia al nacimiento de las malformaciones congénitas en hijos de madres adolescentes con los hijos de madres de entre 20 y 34 años. Período 2002-2011. Hospital Clínico Universidad de Chile. Total de nacimientos del período: 15.636

\begin{tabular}{|c|c|c|c|c|c|}
\hline Diagnóstico & < de 20 años & Tasa/1.000 & 20-34 años & Tasa/1.000 & $\mathbf{p}$ \\
\hline Total de nacimientos por grupo & 1.174 & & 11.063 & & \\
\hline Total de niños con malformaciones & 82 & 70,0 & 968 & 87,5 & 0,04 \\
\hline Agenesia del cuerpo calloso & 1 & 0,9 & 0 & 0,0 & 0,10 \\
\hline Agenesia renal & 0 & 0,0 & 4 & 0,4 & 0,67 \\
\hline Anencefalia & 0 & 0,0 & 6 & 0,6 & 0,54 \\
\hline Ano imperforado & 3 & 2,6 & 9 & 0,8 & 0,10 \\
\hline Aplasia cutis & 2 & 1,7 & 9 & 0,8 & 0,29 \\
\hline Arteria umbilical única & 2 & 1,7 & 23 & 2,1 & 0,56 \\
\hline Artrogriposis & 0 & 0,0 & 3 & 0,3 & 0,74 \\
\hline Atresia de esófago & 0 & 0,0 & 5 & 0,5 & 0,60 \\
\hline Atresia duodenal & 0 & 0,0 & 2 & 0,2 & 0,81 \\
\hline Atresia rectal & 0 & 0,0 & 1 & 0,1 & 0,90 \\
\hline Atresia yeyuno-ileal & 0 & 0,0 & 2 & 0,2 & 0,81 \\
\hline Bridas amnióticas & 0 & 0,0 & 1 & 0,1 & 0,90 \\
\hline Cardiopatía congénita & 8 & 6,8 & 88 & 8,0 & 0,68 \\
\hline Catarata bilateral & 1 & 0,9 & 1 & 0,1 & 0,18 \\
\hline Craneosinostosis & 0 & 0,0 & 2 & 0,2 & 0,81 \\
\hline Criptorquidia & 3 & 2,6 & 32 & 2,9 & 0,56 \\
\hline Diafragma duodenal & 1 & 0,9 & 0 & 0,0 & 0,10 \\
\hline Displasia esquelética & 1 & 0,9 & 5 & 0,5 & 0,45 \\
\hline Displasia renal & 1 & 0,9 & 3 & 0,3 & 0,33 \\
\hline Fístula auris & 3 & 2,6 & 30 & 2,7 & 0,60 \\
\hline Fisura palatina central & 1 & 0,9 & 0 & 0,0 & 0,10 \\
\hline Fosita pilonidal & 7 & 6,0 & 33 & 3,0 & 0,08 \\
\hline Gastrosquisis & 2 & 1,7 & 9 & 0,8 & 0,29 \\
\hline Hemangioma & 7 & 6,0 & 58 & 5,2 & 0,75 \\
\hline Hidronefrosis & 0 & 0,0 & 23 & 2,1 & 0,10 \\
\hline Hidrops no inmunológico & 1 & 0,9 & 2 & 0,2 & 0,26 \\
\hline Hiperplasia suprarrenal congénita & 1 & 0,9 & 2 & 0,2 & 0,26 \\
\hline Holoprosencefalia & 2 & 1,7 & 5 & 0,5 & 0,14 \\
\hline Ictiosis & 1 & 0,9 & 0 & 0,0 & 0,10 \\
\hline Labio Leporino & 0 & 0,0 & 21 & 1,9 & 0,12 \\
\hline
\end{tabular}

No encontramos mayor prevalencia al nacimiento de malformaciones congénitas en los hijos de madres adolescentes comparadas con las madres de 20 a 34 años. En la Tabla 6 se muestra que si bien algunas malformaciones (ano imperforado, fosita pilonidal, pie bot y gastrosquisis) son ligeramente más frecuentes en hijos de madres adolescentes que en hijos de madres de 20 a 34 años estas diferencias no alcanza la significancia estadística.

\section{Discusión}

El primer hecho que llama la atención en nuestra muestra, por ser diferente a lo publicado 
Malformaciones congénitas en hijos de madres adolescentes - J. Nazer et al

Tabla 6 b. Comparación de las tasas de prevalencia al nacimiento de las malformaciones congénitas en hijos de madres adolescentes con los hijos de madres de entre 20 y 34 años. Período 2002-2011. Hospital Clínico Universidad de Chile. Total de nacimientos del período: 15.636

\begin{tabular}{|c|c|c|c|c|c|}
\hline Diagnóstico & $<$ de 20 años & Tasa/1.000 & 20-34 años & Tasa/1.000 & $\mathbf{p}$ \\
\hline Total de nacimientos por grupo & 1.174 & & 11.063 & & \\
\hline Luxación de cadera & 4 & 3,4 & 21 & 2,0 & 0,21 \\
\hline MF Adenomatosa quística & 0 & 0,0 & 4 & 0,4 & 0,67 \\
\hline MF de dedos de los pies & 3 & 2,6 & 11 & 1,0 & 0,15 \\
\hline Microcefalia & 0 & 0,0 & 4 & 0,4 & 0,6 \\
\hline Micrognatia & 0 & 0,0 & 17 & 1,5 & 0,18 \\
\hline Microtia & 2 & 1,7 & 11 & 1,0 & 0,36 \\
\hline Nevo pigmentado & 9 & 7,7 & 145 & 13,1 & 0,11 \\
\hline Nevo sebáceo & 2 & 1,7 & 8 & 0,7 & 0,25 \\
\hline Papiloma preauricular & 4 & 3,4 & 54 & 4,9 & 0,48 \\
\hline Pie baldness & 1 & 0,9 & 2 & 0,2 & 0,26 \\
\hline Pie Bött & 8 & 6,8 & 52 & 4,7 & 0,33 \\
\hline Pielectasia renal & 3 & 2,6 & 20 & 1,8 & 0,38 \\
\hline Polidactilia & 1 & 0,9 & 14 & 1,3 & 0,57 \\
\hline Quiste de ovario & 1 & 0,9 & 6 & 0,6 & 0,51 \\
\hline Secuestro pulmonar & 0 & 0,0 & 5 & 0,5 & 0,60 \\
\hline Sexo ambiguo & 0 & 0,0 & 1 & 0,1 & 0,90 \\
\hline Síndrome de Down & 1 & 0,9 & 17 & 1,5 & 0,47 \\
\hline Síndromes Otros & 1 & 0,9 & 18 & 1,6 & 0,44 \\
\hline Trisomía 18 & 1 & 0,9 & 12 & 1,1 & 0,64 \\
\hline Trisomía 13 & 0 & 0,0 & 4 & 0,4 & 0,67 \\
\hline Triploidía & 0 & 0,0 & 1 & 0,1 & 0,90 \\
\hline Tumores & 0 & 0,0 & 2 & 0,2 & 0,81 \\
\hline Ventrículomegalia & 1 & 0,9 & 4 & 0,4 & 0,40 \\
\hline
\end{tabular}

por nosotros en el año $2003^{7}$, es que el porcentaje de madres adolescentes disminuyó $19,1 \%$ $(\mathrm{p}<0,0001)$, de $9,4 \%$ a $7,6 \%$. Por el contrario el MINSAL da para este grupo etario en el período 1990-2008, un incremento de las frecuencias de $15,6 \%$, desde $13,8 \%$ en 1990 a $16,6 \%$ en el año 2008. Lo observado en el HCUCH es una tendencia que se ha observado desde 1982 a la de fecha, en 1982 el porcentaje de madres menores de 20 años era de 16,08\% y en el año 2011 fue de $7,5 \%$, lo que corresponde a $53,4 \%$ de disminución ( $\mathrm{p}<0,0001)$. Ello demuestra que hay factores socioculturales y económicos diferentes a los de la población de las maternidades de los hospitales del Ministerio de Salud que convendría estudiar, especialmente con el fin de disminuir los embarazos en las adolescentes. La encuesta de caracterización económica (CASEN 2000) encontró que $28,7 \%$ de las niñas entre 14 y 17 años que estaban fuera del sistema escolar obligatorio, se debía principalmente a maternidad y embarazo? Según el Estudio Nacional de Drogas (CONACE), 1 de cada 7 escolares $(15,5 \%)$ consumió una o más veces drogas ilícitas, siendo la marihuana la más frecuentemente utilizada ${ }^{10}$. En nuestra muestra también lo constatamos, pero con frecuencias mayores, $34 \%$ de las madres adolescentes de los niños malformados habían ingerido alcohol antes o durante el embarazo, 39\% fumaban tabaco (39\%) y $18,3 \%$ reconocieron haber fumado marihuana. Si a esto agregamos que cerca de $35 \%$ de estas niñas tenían estudios secundarios completos o estaban estudiando alguna carrera universitaria, tenemos un grupo bastante importante de un nivel cultural 
suficiente como para estar en conocimiento de aspectos de educación sexual.

Existe un hecho que se ha estudiado y comunicado y es la relación que existe entre hábitos de vida de las madres, como consumo de alcohol, drogas y tabaco y la aparición de MFC en sus hijos ${ }^{11-15}$.

El segundo hecho es la importancia de la edad materna como factor de riesgo para MFC. En un trabajo nuestro publicado el año $2007^{16}$, concluíamos que había "una alta asociación entre las mayores prevalencias al nacimiento de MFC y la edad materna, presentándose en una curva en " $U$ " o "J" donde las mayores frecuencias estaban en las madres adolescentes y en las de 35 o más años". En esa muestra la tasa de MFC en los hijos de madres menores de 20 años fue de 9,4\%. Sin embargo, en esta muestra no pudimos demostrar lo mismo, ya que la tasa de prevalencia en los hijos de mujeres adolescentes es de $7 \%$, en circunstancia que la tasa de prevalencia de MFC en hijos de madres entre 20 y 34 años es de $8,8 \%(p=0,04)$.

Al tratar de identificar que anomalías congénitas son más frecuentes en los hijos de este grupo etario, comparado con grupos de madres de mayor edad, encontramos que las tasa de prevalencia de gastrosquisis, pie bot, ano imperforado y fosita pilonidal fueron ligeramente superiores en hijos de madres adolescentes que en hijos de madres entre 20 y 34 años, aunque no alcanzaron la significación estadística $(\mathrm{p}=0,29)$ debido probablemente al bajo número de madres adolescentes en nuestra muestra. Hubo también otras diferencias, no significativas, que se presentan en la Tabla 6 .

\section{Conclusión}

La tasa de prevalencia al nacimiento malformaciones congénitas en hijos de madres adolescentes $(7 \%)$ fue $25,5 \%$ menor $(p=, 009)$ comparada con $9,6 \%$ encontrada en el estudio previo efectuado para el período $1982-2001^{7}$ y es menor a la tasa encontrada en hijos de madres entre 20 y 34 años de edad.

\section{Referencias}

1. www. Minsal.cl (Links).

2. Ramírez C, Nazer J, Cifuentes L, Águila A, Gutiérrez R. Cambios en la distribución etaria de las madres en Chile y en el Hospital Clínico de la Universidad de Chile y su influencia en la morbimortalidad neonatal. Rev Chil Obstet Ginecol 2012; 77 (3): 183-9.

3. Grazi RV, Redheendran R, Mudaliar N, Bannerman RM. Offspring of teenage mothers: congenital malformations, low birth weigths and other finding. J. Reprod Med 1982; 27: 89-96.

4. Vinatier D, Monnier JC, Muller P, Relecour M, Crepin G. Pregnancy and delivery in adolescents. A propos of 122 case reporta. Rev Fr Gynecol Obstet 1984; 79: 549-54.

5. Hutchins FL, Kendall N, Rubino J. Experience with teenage pregnancy. Obstet and Gynecol 1979; 54: 1-5.

6. Larsson J, Svanberg L. Teenage deliveries in Swedish population in 1979's. Acta Obstet Gynecol Scand 1983; 62: 467-72.

7. Pardo RA, Nazer J, Cifuentes L. Prevalencia al nacimiento de malformaciones congénitas y de menor peso de nacimiento en hijos de madres adolescentes. Rev Med Chile 2003; 131: 1165-72.

8. Castilla EE. Manual Operacional del Estudio Colaborativo Latino Americano de Malformaciones Congénitas (ECLAMC). 1995. Fio Cruz. Río de Janeiro. Ed Fundación Oswaldo Cruz.

9. Ministerio de Educación de la República de Chile. Encuesta de caracterización socioeconómica nacional (CASEN 2000) sobre el estado de la educación en Chile. 2001. (Links).

10. Ministerio del Interior de la República de Chile. CONACE. Estudio nacional de drogas en la población escolar de Chile. 2001. (Links).

11. Morales-Suárez M, Mille C, Christensen K. Smoking habits, Nicotina Use and Congenital Malformations. Obstet Gynecol 2006; 107: 51-7.

12. Bianchi F, Calzolari E, Hermien EK, Knill-Jones R, and the Occupational Exposure and Congenital Malformation Working Groupe. Am J Public Health. 2000.

13. Baumann P, Schild C, Hume RF, Sokol RJ. Alcohol abuse. A persistent preventable risk for congenital Anomalies. International Journal of Gynecology and Obstetrics (2006) 95, 66-72.

14. Woods S, Raju U. Maternal Smoking and the risk of Congenital Birth Defects: A Cohort Study. JABFP September-october 2001; Vol. 14 No. 5.

15. Behnke M, Davis Eyler F, Wilson C, Wobie K. The search for congenital Malformations in Newborns with fetal cocaine. Pediatrics 2001; 107: 74

16. Nazer J, Cifuentes L, Aguila A, Ureta P, Bello MP, Correa F, Melibosky R. Edad materna y malformaciones congénitas. Un Registro de 35 años. 1970-2005. Rev Med Chile 2007; 135: 1463-69. 